\title{
Prediction of seed gene function in progressive diabetic neuropathy by a network-based inference method
}

\author{
SHAN-SHAN LI ${ }^{1}$, XIN-BO ZHAO ${ }^{1}$, JIA-MEI TIAN ${ }^{2}$, HAO-REN WANG $^{3}$ and TONG-HUAN WEI ${ }^{4}$ \\ Departments of ${ }^{1}$ Endocrinology and ${ }^{2}$ Pediatrics, Linyi People's Hospital; ${ }^{3}$ Department of Medicine, \\ Linyi Luozhuang Central Hospital; ${ }^{4}$ Department of Medicine, People's Hospital \\ of Linyi High-Tech Industrial Development Zone, Linyi, Shandong 276000, P.R. China
}

Received July 6, 2018; Accepted March 7, 2019

DOI: $10.3892 /$ etm.2019.7441

\begin{abstract}
Guilt by association (GBA) algorithm has been widely used to statistically predict gene functions, and network-based approach increases the confidence and veracity of identifying molecular signatures for diseases. This work proposed a network-based GBA method by integrating the GBA algorithm and network, to identify seed gene functions for progressive diabetic neuropathy (PDN). The inference of predicting seed gene functions comprised of three steps: i) Preparing gene lists and sets; ii) constructing a co-expression matrix (CEM) on gene lists by Spearman correlation coefficient (SCC) method and iii) predicting gene functions by GBA algorithm. Ultimately, seed gene functions were selected according to the area under the receiver operating characteristics curve (AUC) index. A total of 79 differentially expressed genes (DEGs) and 40 background gene ontology (GO) terms were regarded as gene lists and sets for the subsequent analyses, respectively. The predicted results obtained from the network-based GBA approach showed that $27.5 \%$ of all gene sets had a good classified performance with AUC $>0.5$. Most significantly, 3 gene sets with AUC $>0.6$ were denoted as seed gene functions for PDN, including binding, molecular function and regulation of the metabolic process. In summary, we predicted 3 seed gene functions for PDN compared with non-progressors utilizing network-based GBA algorithm. The findings provide insights to reveal pathological and molecular mechanism underlying PDN.
\end{abstract}

Correspondence to: Dr Tong-Huan Wei, Department of Medicine, People's Hospital of Linyi High-Tech Industrial Development Zone, 135 Huoju Road, High-Tech Zone, Luo-Zhuang, Linyi, Shandong 276000, P.R. China

E-mail: tonghuanweily@163.com

Key words: co-expression, gene function, guilt by association, network, progressive diabetic neuropathy

\section{Introduction}

Diabetic neuropathy (DN) is the most common and troublesome complication of diabetes, characterized by the gradual loss of peripheral axons, resulting in diminished sensation, pain, and eventual complete loss of sensation (1). Pathologically, it is the culprit of a series of interrelated metabolic abnormalities with insulin deficiency and hyperglycemia (2). Importantly, DN affects up to $60-70 \%$ of diabetics, leading to the highest morbidity and mortality rates and to a huge economic burden of diabetes care $(3,4)$. However, in addition to controlling blood glucose levels, no effective treatment options have been found to prevent, slow or reverse the progression of DN and are not always achievable even in alert patients (5). Besides, a good understanding of pathological and molecular mechanism underlying DN might give help to explore effective therapy of this complicated disease.

The difference of gene expression levels could reflect the propensity of many diseases, and thus identifying gene functions has been an effective way to reveal the pathological mechanism of a disease at molecular level (6). ELAVL3, as a member of the Elavl family, is known as a neuronal Elavl because it is expressed in peripheral and central neurons throughout development (7). Ogawa et al hold the view that ELAVL3 is essential for maintaining Purkinje neuron axons (8). It has been reported that ELAVL3 regulates neuronal polarity via alternative splicing of embryo-specific exon in AnkyrinG (9). There is some evidence to suggest that Molecule Interacting with CasL-Likel (MICALL1), involved in pre-cytokinetic events, acts as a membrane hub on tubular recycling endosomes (10). According to recent reports, tubular recirculating endosome biogenesis was regulated through p53-MICALL1 pathway (11). HEY2, as a member of hairy-related basic helix-loop-helix transcription factor subfamily, is involved in boundary formation and cell fate determination (12). Recent research has indicated that miR-98 activated the Notch signaling pathway by binding to HEY2 in Alzheimer's disease mice to improve mitochondrial dysfunction and oxidative stress (13). Our research results agree with previous studies, suggesting that ELAVL3, MICALL1, HEY2 genes have effects on neurological diseases. It has been demonstrated that by using variants based on the guilty susceptibility (GBA) algorithm, gene function prediction can 
be performed with very high statistical confidence assuming that the associations in the genetic data is necessary to establish culpability (14). Although various of techniques have been proposed for purpose of extending the GBA to indirect connections, only slight effectiveness has been discovered (15-18).

In the present study, a new method was proposed to predict seed gene functions for progressive DN (PDN) patients, by integrating the GBA algorithm and network-based method. To achieve this goal, gene expression data and gene ontology (GO) annotations were collected from the public databases. Differentially expressed genes (DEGs) were identified as gene lists and background GO terms were extracted as gene sets. The co-expression matrix (CEM) was constructed on gene lists by Spearman correlation coefficient (SCC) method.

\section{Materials and methods}

Preparing gene expression data. Gene expression data [GSE24290 (19)] for human DN were recruited from the public-free Gene Expression Omnibus (GEO). In brief, based on the density of myelinated fibers, GSE24290 divided the DN patient samples into two groups, progressors and non-progressors. In short, the patients in the progressive group lost $\geq 500$ fibres $/ \mathrm{mm}^{2}$, and the patients in the non-progressive group lost $\leq 100$ fibres $/ \mathrm{mm}^{2}$ over 52 weeks (20). By mapping these preprocessed probes to gene structures, a total of 10,570 genes were identified for PDN for subsequent analysis.

Collecting gene sets. All human GO annotations were prepared from the Gene Ontology Consortium $(21,22)$. For purpose of making these retained GO terms more correlated to progressors, we took the intersections between DEGs and GO terms. If the number of DEGs for a GO term was smaller than 20, it was removed. Only GO terms including equal or more than 20 DEGs were reserved.

Identifying DEGs. DEGs between progressors and non-progressors were detected (23). The lmFit function implemented in Limma was utilized to perform linear fitting, empirical Bayes statistics and false discovery rate (FDR) calibration of the P-values on the data $(24,25)$. The thresholds for DEGs were set as $\mathrm{P}<0.05$ and $\log _{2}$ fold-changel $>2$.

Constructing CEM. To further investigate the correlations or interactions among DEGs, a CEM for them was constructed based on the SCC algorithm (26). If the SCC for a pair of genes was positive, it would indicate a positive linear correlation between the two genes. Similarly, a negative SCC refers to a negative relationship of the gene pair. In addition, the absolute SCC value of an interaction was denoted as its weight value. Furthermore, the higher the weight value across two genes, the stronger the interaction was, especially for 1 . Otherwise, the 0 meant that there was no interaction between two genes. As a result, a CEN was constructed according to weight.

Network-based GBA algorithm. The GBA algorithm was combined with network to predict significant gene functions for progressors. Specifically, for a DEG in the CEM, we chose its adjacent genes to enrich to a GO term. Based on these GO functional annotations, a multi-functionality (MF) score was assigned to each gene $i$ in the CEM (14),

$$
S(i)=\sum_{k \vee i \in G O_{k}} \frac{1}{\operatorname{Num}_{\text {in }_{k}} * \text { Num }_{\text {out }_{k}}}
$$

of which $\mathrm{Num}_{i n_{k}}$ represented the number of genes within GO group $k$, whose weighting had the effect of giving contribution to a GO group; and $\mathrm{Num}_{\text {out }_{k}}$ was the number of genes outside GO group $k$ in the CEM, whose weighting provided a corresponding weighting to genes not within the GO group. Note that we computed the AUC for evaluating classification performances between progressors and non-progressors. Here, for assessing the predictive power of machine learners in the support vector machine (SVM) model, AUC is an assessment of the accuracy of clinical classification performance (27). Most importantly, an AUC of 0.5 represents classification at chance levels, while an AUC of 1.0 represents a perfect classification. Thus we defined GO terms of AUC $>0.6$ as seed gene functions for PDN patients in the present report.

\section{Results}

Gene lists. In this report, based on 10,570 genes in GSE24290, we identified 79 DEGs between progressors and non-progressors by Limma package when setting the thresholds as $\mathrm{P}<0.05$ and $\log _{2}$ fold-changel $>2$. All DEGs were ranked in ascending order of their P-values (Table I). We found that the most significant DEGs were ELAVL3 ( $\mathrm{P}=1.87 \mathrm{E}-02)$, MICALL1 ( $\mathrm{P}=2.51 \mathrm{E}-02)$, HEY2 (P=3.42E-02), PCDHB1 ( $\mathrm{P}=3.69 \mathrm{E}-02)$ and OR2S2 (P=4.81E-02). Importantly, the 79 DEGs were regarded as gene lists for further exploitation.

Gene sets. In the Gene Ontology Consortium database, there are 19,003 gene sets involved in 18,402 genes. To make these sets with a stable performance and more correlated to progressors, we chose GO terms from 20 to 1,000 in size and then took intersections between the reserved terms with the gene lists. Only gene sets containing intersected DEGs $>20$ were left in the subsequent analysis. Hereinafter, we defined the amount of intersected DEGs as the count value of this term. As a result, a total of $40 \mathrm{GO}$ terms were determined (Table II), termed with background GO terms. There were three terms with Count $>60$, cellular component $(\mathrm{GO}: 0005575$, Count $=71)$, biological process (GO:0008150, Count $=67)$, and molecular function $(\mathrm{GO}: 0003674$, Count $=66)$. Furthermore, we calculated the DEGs by expressing the spectral data. According to the GO enrichment analysis, the enrichment of 79 DEGs in background GO terms was obtained (28). DEG annotated to a GO term, and if the gene is present in the GO term, the value is 1 (red), otherwise 0 (yellow). Finally, the heatmap was obtained from the heatmap package in the $\mathrm{R}$ language to analyze the above enrichment situation (Fig. 1). The result of this heatmap was in accordance with Table II. Ultimately, the background GO terms were the gene sets used.

CEM. With an attempt to investigate biological correlations among DEGs, a CEM with 79 nodes and 3,081 interactions were constructed based on the SCC, of which each interaction possessed a weight value to reveal the interacted strength 
Table I. Gene list for progressive diabetic neuropathy (PDN).

\begin{tabular}{|c|c|c|c|c|c|}
\hline Rank & DEG & P-value & Rank & DEG & P-value \\
\hline 1 & ELAVL3 & 0.018730149 & 41 & FER1L4 & 0.237218317 \\
\hline 2 & MICALL1 & 0.025064168 & 42 & TAS2R13 & 0.254492341 \\
\hline 3 & HEY2 & 0.034183011 & 43 & SYN1 & 0.254519864 \\
\hline 4 & PCDHB1 & 0.036938421 & 44 & KCNMB4 & 0.257387331 \\
\hline 5 & OR2S2 & 0.048112652 & 45 & SLC35E1 & 0.258472445 \\
\hline 6 & CNTD2 & 0.055977842 & 46 & PRX & 0.267184163 \\
\hline 7 & DNAJB12 & 0.059213903 & 47 & GIPC2 & 0.276805505 \\
\hline 8 & GPR21 & 0.061091712 & 48 & RBM12B & 0.298659702 \\
\hline 9 & PFN1 & 0.068351436 & 49 & HACD1 & 0.302339336 \\
\hline 10 & ZMIZ2 & 0.073944462 & 50 & NAALAD2 & 0.309726354 \\
\hline 11 & HSPA6 & 0.080467794 & 51 & KLHL21 & 0.310864132 \\
\hline 12 & TRIM36 & 0.082922925 & 52 & TMX2 & 0.316649651 \\
\hline 13 & MTHFD1 & 0.08313726 & 53 & HEBP2 & 0.319262815 \\
\hline 14 & RPA4 & 0.091277628 & 54 & RFC2 & 0.319345713 \\
\hline 15 & NIPAL2 & 0.101024558 & 55 & CCDC134 & 0.327623954 \\
\hline 16 & HDDC2 & 0.106181304 & 56 & MEPE & 0.343481838 \\
\hline 17 & LIME1 & 0.107101383 & 57 & TRAPPC9 & 0.374991751 \\
\hline 18 & MAPKAPK5-AS1 & 0.108867691 & 58 & TXNRD1 & 0.384530053 \\
\hline 19 & CCDC186 & 0.116573636 & 59 & CTDNEP1 & 0.386873156 \\
\hline 20 & FCF1 & 0.117569875 & 60 & SH2D4A & 0.39096557 \\
\hline 21 & PSMD5 & 0.118641952 & 61 & ZNF512B & 0.390996512 \\
\hline 22 & YTHDF3 & 0.130853807 & 62 & TAGLN2 & 0.400497742 \\
\hline 23 & CDHR5 & 0.135952991 & 63 & C10orf76 & 0.401347031 \\
\hline 24 & C6orf120 & 0.1416115 & 64 & PIK3IP1 & 0.409666004 \\
\hline 25 & VWA7 & 0.144178178 & 65 & C5orf30 & 0.420802441 \\
\hline 26 & ASF1A & 0.147208457 & 66 & PKNOX1 & 0.449555958 \\
\hline 27 & NELL2 & 0.162233252 & 67 & DNAJB2 & 0.465398033 \\
\hline 28 & SRM & 0.167545586 & 68 & NCR2 & 0.474076273 \\
\hline 29 & SH3KBP1 & 0.176459493 & 69 & GALNT8 & 0.475841943 \\
\hline 30 & USE1 & 0.178993287 & 70 & IKZF3 & 0.480703371 \\
\hline 31 & INSIG1 & 0.179397171 & 71 & SENP5 & 0.492341515 \\
\hline 32 & SSH3 & 0.18328187 & 72 & POMGNT2 & 0.510089318 \\
\hline 33 & FAM66D & 0.185333874 & 73 & CALHM2 & 0.515258516 \\
\hline 34 & PGAP3 & 0.190879424 & 74 & ATP8B4 & 0.5305693 \\
\hline 35 & PER2 & 0.192655455 & 75 & GALNT10 & 0.543825962 \\
\hline 36 & CCS & 0.193106321 & 76 & WIZ & 0.550465591 \\
\hline 37 & E2F8 & 0.200074614 & 77 & FER & 0.594006462 \\
\hline 38 & HTR1F & 0.221840324 & 78 & PITPNA & 0.597706177 \\
\hline 39 & ZNF142 & 0.232301069 & 79 & MYO3A & 0.599378182 \\
\hline 40 & PPP1R3B & 0.236879137 & & & \\
\hline
\end{tabular}

between two genes. The weight distribution for interactions in this CEM showed the characteristic of good adjacent matrix that weights on its diagonal nearly equaled to 1 , which suggested that the CEM had a good network scale property. In particular, an edge between C6orf120 and PGAP3 had the highest weight of 0.994. Moreover, to further evaluate the activities of genes in interactions of high weights, topological degree centrality analysis was conducted on all nodes in the CEM. The results showed that C6orf120 connected with 56 adjacent genes and thus had the highest degree. Subsequently, an assortativity coefficient was calculated to assess degree assortative mixing pattern extent. Consequently, the assortativity coefficient for the CEM was 0.829 , indicating that the network had perfect assortative mixing patterns.

A sub-network was extracted from the CEM by selecting those interactions with weight $>0.8$ and visualized it by Cytoscape software (Fig. 2). There were 48 nodes and 330 edges in the sub-network. Among these nodes, PGAP3, C6orf120 and RBM12B had higher degree than the others, which suggested their key roles in the PCN patients and 


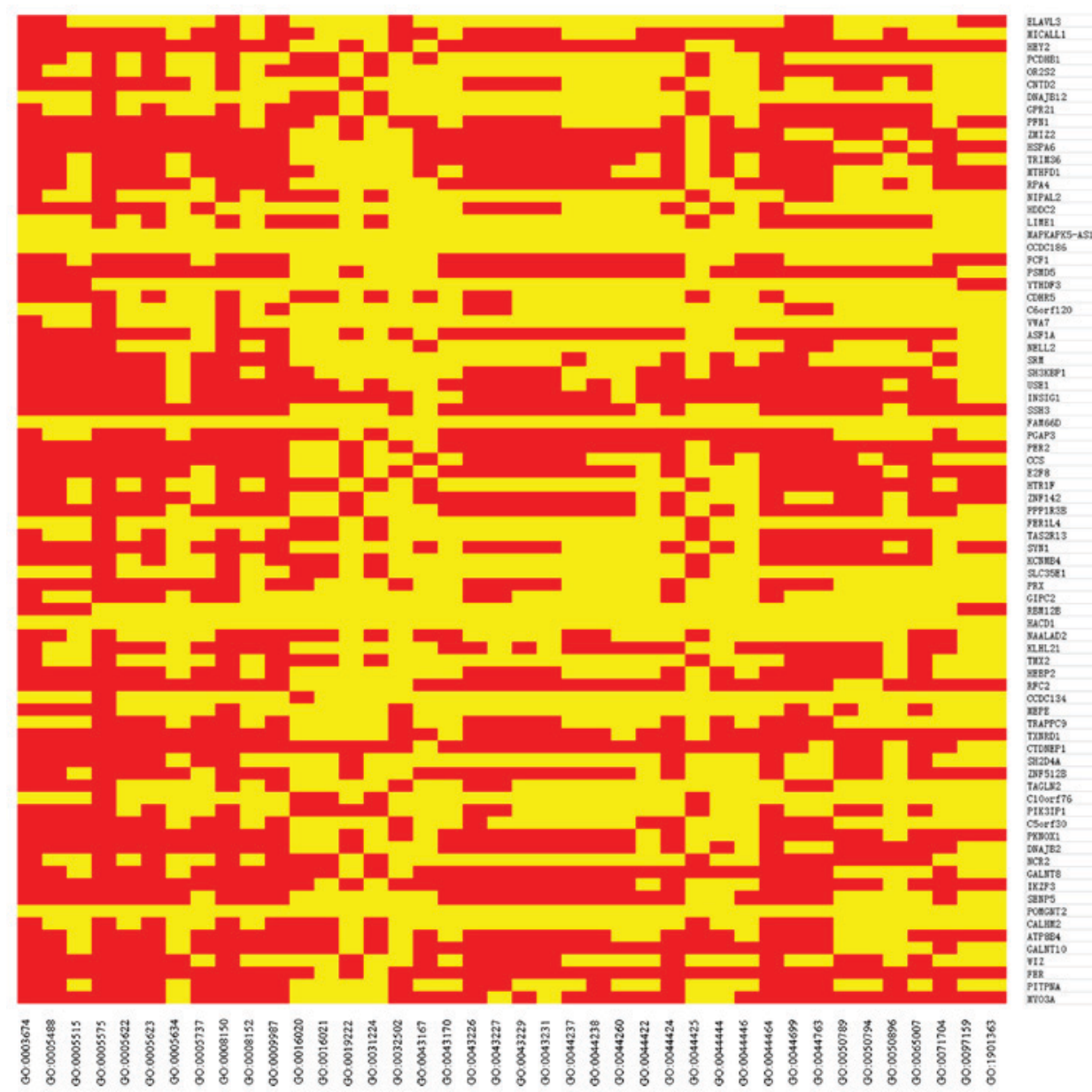

Figure 1. Heatmap for gene lists and sets. The ordinate, 79 differentially expressed genes (DEGs), the abscissa the 40 GO terms. Red, the association between the gene and the go term in sample channel. Yellow, no correlation between the gene and the go term in reference channel.

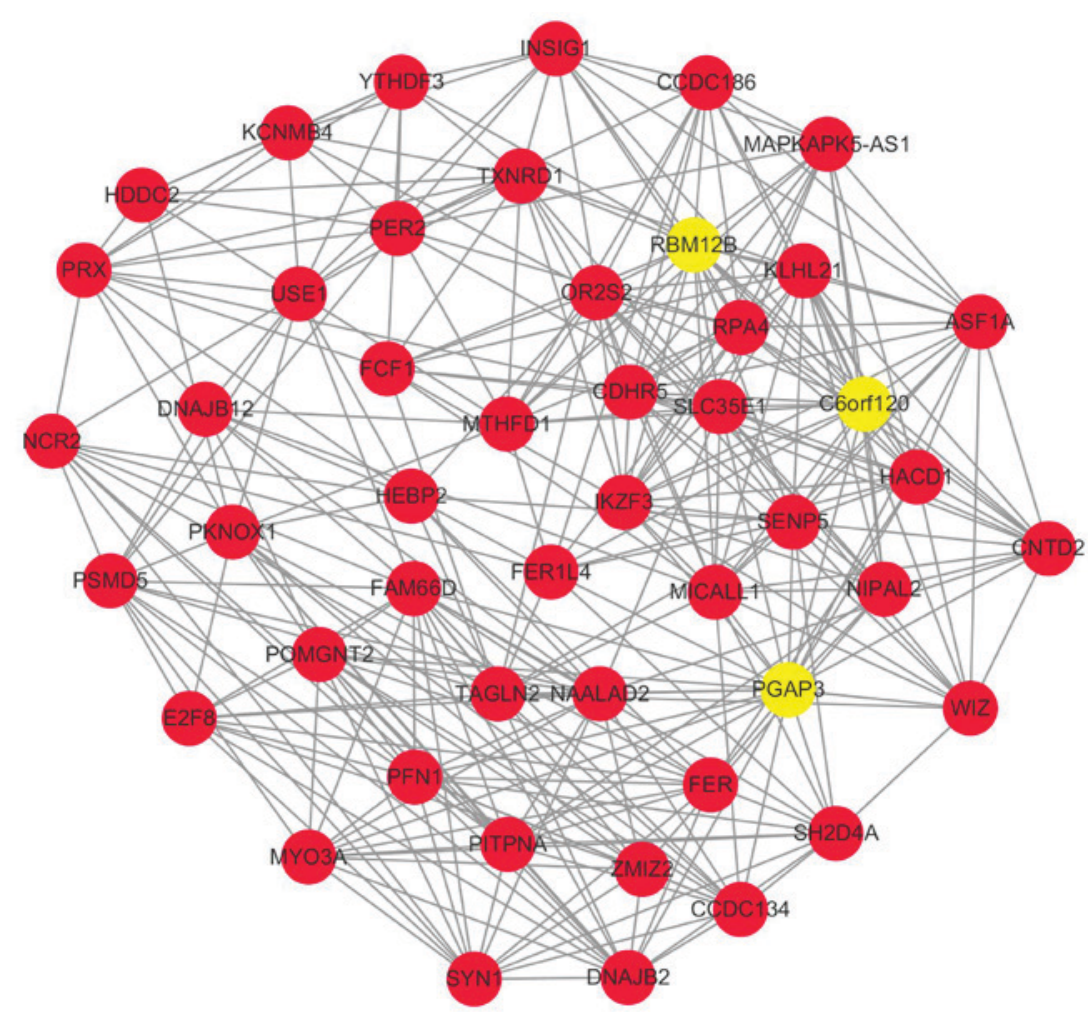

Figure 2. Sub-network of co-expression matrices (CEM). There were 48 nodes and 330 edges, of which the nodes were differentially expressed genes (DEGs) and the edges interactions between two DEGs with weight $>0.8$. 
Table II. Gene sets for progressive diabetic neuropathy (PDN).

\begin{tabular}{llcllc}
\hline GO ID & \multicolumn{1}{c}{ GO term } & Counts & GO ID & & GO term \\
\hline GO:0005575 & Cellular component & 71 & GO:0008152 & Metabolic process & 36 \\
GO:0008150 & Biological process & 67 & GO:0071704 & Organic substance metabolic process & 34 \\
GO:0003674 & Molecular function & 66 & GO:0016020 & Membrane & 33 \\
GO:0005623 & Cell & 58 & GO:0044237 & Cellular metabolic process & 33 \\
GO:0044464 & Cell part & 58 & GO:0044238 & Primary metabolic process & 33 \\
GO:0009987 & Cellular process & 57 & GO:0043170 & Macromolecule metabolic process & 28 \\
GO:0005488 & Binding & 55 & GO:0044425 & Membrane part & 27 \\
GO:0044699 & Single-organism process & 52 & GO:0044444 & Cytoplasmic part & 27 \\
GO:0043226 & Organelle & 49 & GO:0044260 & Cellular macromolecule metabolic process & 26 \\
GO:0044763 & Single-organism cellular process & 49 & GO:0005634 & Nucleus & 25 \\
GO:0005622 & Intracellular & 47 & GO:0016021 & Integral component of membrane & 25 \\
GO:0044424 & Intracellular part & 47 & GO:0031224 & Intrinsic component of membrane & 25 \\
GO:0043227 & Membrane-bounded organelle & 46 & GO:0043167 & Ion binding & 24 \\
GO:0065007 & Biological regulation & 43 & GO:0050896 & Response to stimulus & 24 \\
GO:0043229 & Intracellular organelle & 42 & GO:0044422 & Organelle part & 23 \\
GO:0005515 & Protein binding & 41 & GO:0097159 & Organic cyclic compound binding & 23 \\
GO:0043231 & Intracellular membrane-bounded & 40 & GO:1901363 & Heterocyclic compound binding & 23 \\
& organelle & & & & 22 \\
GO:0050789 & Regulation of biological process & 39 & GO:0044446 & Intracellular organelle part & 21 \\
GO:0005737 & Cytoplasm & 38 & GO:0019222 & Regulation of metabolic process & 21 \\
GO:0050794 & Regulation of cellular process & 37 & GO:0032502 & Developmental process & \\
\hline
\end{tabular}

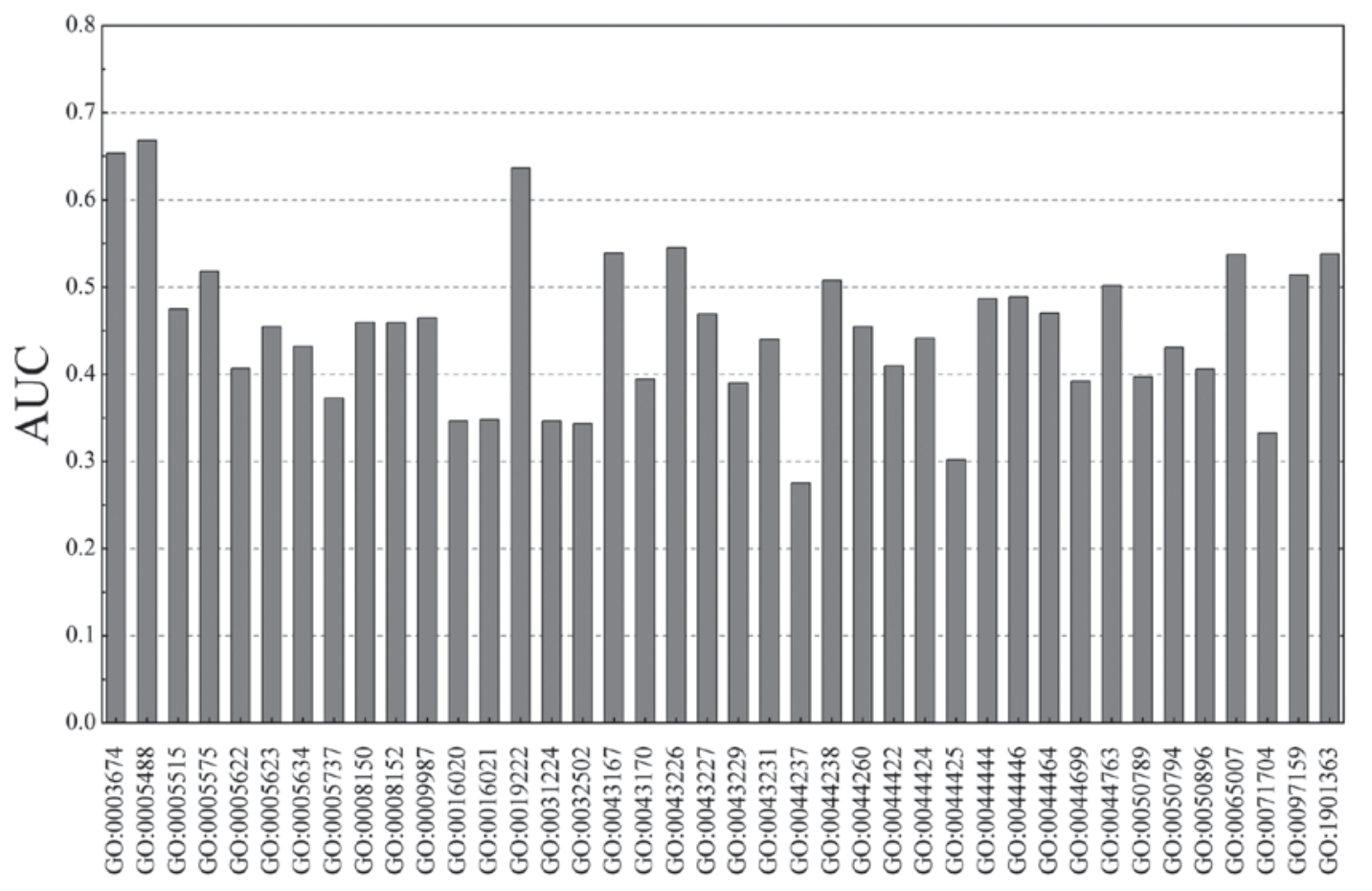

Figure 3. The area under the receiver operating characteristics curve (AUC) distribution for the gene sets.

inferred that gene functions participated by them might act as critical processes in progressors.

Seed gene functions. The AUC index was applied to evaluate the classified performance on MF scores between progressors and non-progressors by the 3 -fold cross-validation. Generally, if an AUC for a gene or process was $>0.5$, it could be used to classify the case group from controls. In this report, the AUC for the 40 gene sets are shown in Fig. 3. There were $11 \mathrm{GO}$ terms with AUC $>0.5$ in total. Thus, 11 of 40 (27.5\%) gene sets 
had a good classification performance between progressors and non-progressors of DN. Among them, 3 with AUC >0.6 were considered to be seed gene functions in progressors of DN. The seed gene functions were Binding (GO:0005488, $\mathrm{AUC}=0.668)$, Molecular function $(\mathrm{GO}: 0003674, \mathrm{AUC}=0.654)$, and Regulation of metabolic process (GO:0019222, $\mathrm{AUC}=0.636$ ).

\section{Discussion}

In the present study, we predicted seed gene functions in PDN patients by using a network-based GBA method, since the network-based approach systematically investigate the molecular complexity of a particular disease (29) and identify potential signatures through bio-molecular networks rather than individual genes $(30,31)$. An integration of co-expression network and the GBA algorithm provide a new manner to predict significant gene functions and reveal molecular mechanism underlying PDN.

On the basis of SCC method, a CEM for progressors was constructed on DEGs, and a sub-network of weight $>0.8$ was extracted from the CEM. Interestingly, we found that $P G A P 3$, C6orf120 and RBM12B had high degree both in CEM and its sub-network, which indicated their importance in progressors. Taking $R B M 12 B$ as an example, $R B M 12 B$ (RNA binding motif protein $12 \mathrm{~B}$ ) is a protein coding gene that relates to functions of RNA binding, nucleic acid binding and nucleotide binding (32). It has been demonstrated that $R b m 12 b$ and $R b m 3$ are mainly down-regulated and highly responsive to systemic hypoxia in mouse developing brain and placenta (33). This is the first time the key role of $R B M 12 B$ in human PDN patients was uncovered. In addition, in our study, this gene was enriched in two seed gene functions, Binding and Molecular function. The possible inference was that the dys-regulation of $R B M 12 B$ might disturb the normal functions of binding and lead to brain injury, even lesions of the nervous system.

Particularly, a total of 40 background GO terms were identified as gene sets for the current study. Subsequently, an MF score was assigned to each gene in the specific gene set, and then an AUC for each GO term was produced to assess the prediction performance between progressors and non-progressors. In consequence, $27.5 \%$ of all gene sets had a good classified performance with AUC $>0.5$. Most significantly, 3 gene sets with AUC $>0.6$ were denoted as seed gene functions for PDN, including Binding, Molecular function and Regulation of metabolic process. It is a common phenomenon that a given gene is present in one or more molecular functions (such as RBM12B described above), and two or more genes exhibit the same function.

Cameron et al reviewed that poor metabolic control was one of microvascular complications correlated to DN (34). Thus, it was important to improve the metabolic conditions that led to the pathology underlying peripheral DN, and the metabolic correction modified symptom control and clinical results (35). Moreover, metabolic dysfunction in experimental DN due to energy homeostasis and/or oxidative stress is limited to the sciatic nerve (36). Above all, we might conclude various metabolic processes play crucial roles in DP, and thus the regulation of metabolic process has become informative in PDN patients.
In summary, we have predicted 3 seed gene functions for progressors of DP compared with non-progressors utilizing network-based GBA algorithm. The findings provide insights to reveal pathological and molecular mechanism underlying PDN. However, the expression data used in this work was recruited from the open access database, and the 3 seed gene functions still need to be validated.

\section{Acknowledgements}

Not applicable.

\section{Funding}

No funding was received.

\section{Availability of data and materials}

The datasets used and/or analyzed during the current study are available from the corresponding author on reasonable request.

\section{Authors' contributions}

SSL and XBZ conceived the study and drafted the manuscript. XBZ, JMT and HRW acquired the data. SSL and THW analyzed the data and revised the manuscript. All the authors read and approved the final manuscript.

\section{Ethics approval and consent to participate}

Not applicable.

\section{Patient consent for publication}

Not applicable.

\section{Competing interests}

The authors declare that they have no competing interests.

\section{References}

1. Edwards JL, Vincent AM, Cheng HT and Feldman EL: Diabetic neuropathy: Mechanisms to management. Pharmacol Ther 120: $1-34,2008$

2. Sima AAF and Zhang W: Mechanisms of diabetic neuropathy: Axon dysfunction. Handb Clin Neurol 126: 429-442, 2014.

3. Vinik AI, Nevoret ML, Casellini C and Parson H: Diabetic neuropathy. Endocrinol Metab Clin North Am 42: 747-787, 2013.

4. Oyenihi AB, Ayeleso AO, Mukwevho E and Masola B Antioxidant strategies in the management of diabetic neuropathy. BioMed Res Int 2015: 515042, 2015.

5. Charnogursky G, Lee H and Lopez N: Diabetic neuropathy. Handb Clin Neurol 120: 773-785, 2014.

6. Zhao J, Yang TH, Huang Y and Holme P: Ranking candidate disease genes from gene expression and protein interaction: A Katz-centrality based approach. PLoS One 6: e24306, 2011.

7. Good PJ: The role of elav-like genes, a conserved family encoding RNA-binding proteins, in growth and development. Semin Cell Dev Biol 8: 577-584, 1997.

8. Ogawa Y, Kakumoto K, Yoshida T, Kuwako KI, Miyazaki T, Yamaguchi J, Konno A, Hata J, Uchiyama Y, Hirai $\mathrm{H}$, et al: Elavl3 is essential for the maintenance of Purkinje neuron axons. Sci Rep 8: 2722, 2018. 
9. Ogawa Y, Yamaguchi J, Yano M, Uchiyama Y and Okano HJ: Elav13 regulates neuronal polarity through the alternative splicing of an embryo-specific exon in AnkyrinG. Neurosci Res 135: 13-20, 2018.

10. Reinecke JB, Katafiasz D, Naslavsky N and Caplan S: Novel functions for the endocytic regulatory proteins MICAL-L1 and EHD1 in mitosis. Traffic 16: 48-67, 2015.

11. Takahashi Y, Tanikawa C, Miyamoto T, Hirata M, Wang G, Ueda K, Komatsu T and Matsuda K: Regulation of tubular recycling endosome biogenesis by the p53-MICALL1 pathway. Int J Oncol 51: 724-736, 2017.

12. Miao L, Li J, Li J, Tian X, Lu Y, Hu S, Shieh D, Kanai R, Zhou BY, Zhou B, et al: Notch signaling regulates Hey2 expression in a spatiotemporal dependent manner during cardiac morphogenesis and trabecular specification. Sci Rep 8: 2678, 2018.

13. Chen FZ, Zhao Y and Chen HZ: MicroRNA-98 reduces amyloid $\beta$-protein production and improves oxidative stress and mitochondrial dysfunction through the Notch signaling pathway via HEY2 in Alzheimer's disease mice. Int J Mol Med 43: 91-102, 2019.

14. Gillis $\mathrm{J}$ and Pavlidis P: The impact of multifunctional genes on 'guilt by association' analysis. PLoS One 6: e17258, 2011.

15. Chua HN, Sung WK and Wong L: Exploiting indirect neighbours and topological weight to predict protein function from protein-protein interactions. Bioinformatics 22: 1623-1630, 2006

16. Yip AM and Horvath S: Gene network interconnectedness and the generalized topological overlap measure. BMC Bioinformatics 8 : 22, 2007.

17. Weston AD and Hood L: Systems biology, proteomics, and the future of health care: Toward predictive, preventative, and personalized medicine. J Proteome Res 3: 179-196, 2004.

18. Peña-Castillo L, Tasan M, Myers CL, Lee H, Joshi T, Zhang C Guan Y, Leone M, Pagnani A, Kim WK, et al: A critical assessment of Mus musculus gene function prediction using integrated genomic evidence. Genome Biol 9 (Suppl 1): S2, 2008

19. Hur J, Sullivan KA, Pande M, Hong Y, Sima AA, Jagadish HV, Kretzler $M$ and Feldman EL: The identification of gene expression profiles associated with progression of human diabetic neuropathy. Brain 134: 3222-3235, 2011

20. Wiggin TD, Sullivan KA, Pop-Busui R, Amato A, Sima AAF and Feldman EL: Elevated triglycerides correlate with progression of diabetic neuropathy. Diabetes 58: 1634-1640, 2009.

21. Consortium GO; Gene Ontology Consortium: Gene Ontology Consortium: Going forward. Nucleic Acids Res 43 (D1): D1049-D1056, 2015.

22. Gillis $\mathrm{J}$ and Pavlidis P: The role of indirect connections in gene networks in predicting function. Bioinformatics 27: 1860-1866, 2011.

23. Ritchie ME, Phipson B, Wu D, Hu Y, Law CW, Shi W and Smyth GK: limma powers differential expression analyses for RNA-sequencing and microarray studies. Nucleic Acids Res 43: e47, 2015
24. Datta S, Satten GA, Benos DJ, Xia J, Heslin MJ and Datta S: An empirical bayes adjustment to increase the sensitivity of detecting differentially expressed genes in microarray experiments. Bioinformatics 20: 235-242, 2004.

25. Reiner A, Yekutieli D and Benjamini Y: Identifying differentially expressed genes using false discovery rate controlling procedures. Bioinformatics 19: 368-375, 2003.

26. Szmidt E and Kacprzyk J: The Spearman rank correlation coefficient between intuitionistic fuzzy sets. In: 2010 5th IEEE International Conference Intelligent Systems. IEEE, London, pp276-280, 2010.

27. Huang $\mathrm{J}$ and Ling $\mathrm{CX}$ : Using AUC and accuracy in evaluating learning algorithms. Knowledge and Data Engineering. IEEE Trans 17: 299-310, 2005

28. Zhu Q, Sun Y, Zhou Q, He Q and Qian H: Identification of key genes and pathways by bioinformatics analysis with TCGA RNA sequencing data in hepatocellular carcinoma. Mol Clin Oncol 9: 597-606, 2018

29. Barabási AL, Gulbahce N and Loscalzo J: Network medicine: A network-based approach to human disease. Nat Rev Genet 12: 56-68, 2011.

30. Liu ZP, Wang Y, Zhang XS and Chen L: Network-based analysis of complex diseases. IET Syst Biol 6: 22-33, 2012.

31. Chen L, Wang RS and Zhang XS: Reconstruction of gene regulatory networks. In: Biomolecular Networks: Methods and Applications in Systems Biology. John Wiley \& Sons, Inc., Hoboken, NJ, pp47-87, 2009.

32. Zhang G, Bowling H, Hom N, Kirshenbaum K, Klann E, Chao MV and Neubert TA: In-depth quantitative proteomic analysis of de novo protein synthesis induced by brain-derived neurotrophic factor. J Proteome Res 13: 5707-5714, 2014.

33. Trollmann R, Rehrauer H, Schneider C, Krischke G, Huemmler N, Keller S, Rascher W and Gassmann M: Late-gestational systemic hypoxia leads to a similar early gene response in mouse placenta and developing brain. Am J Physiol Regul Integr Comp Physiol 299: R1489-R1499, 2010.

34. Cameron NE, Eaton SEM, Cotter MA and Tesfaye S: Vascular factors and metabolic interactions in the pathogenesis of diabetic neuropathy. Diabetologia 44: 1973-1988, 2001.

35. Miranda-Massari JR, Gonzalez MJ,Jimenez FJ, Allende-Vigo MZ and Duconge J: Metabolic correction in the management of diabetic peripheral neuropathy: Improving clinical results beyond symptom control. Curr Clin Pharmacol 6: 260-273, 2011.

36. Freeman OJ,Unwin RD,Dowsey AW,BegleyP,AliS,Hollywood KA, Rustogi N, Petersen RS, Dunn WB, Cooper GJ, et al: Metabolic dysfunction is restricted to the sciatic nerve in experimental diabetic neuropathy. Diabetes 65: 228-238, 2016.

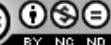

This work is licensed under a Creative Commons Attribution-NonCommercial-NoDerivatives 4.0 International (CC BY-NC-ND 4.0) License. 